\title{
L'impact des trajectoires familiales sur les parcours résidentiel et géographique des franciliens nés entre 1930 et 1950
}

The impact of family histories on the residential and geographical trajectories of residents of the Paris region born between 1930 and 1950

Catherine Bonvalet et Arnaud Bringé

\section{OpenEdition \\ Journals}

Édition électronique

URL : http://journals.openedition.org/ress/3452

DOI : $10.4000 /$ ress.3452

ISBN : $1663-4446$

ISSN : 1663-4446

Éditeur

Librairie Droz

Édition imprimée

Date de publication : 15 mai 2016

Pagination : 75-102

ISSN : 0048-8046

Référence électronique

Catherine Bonvalet et Arnaud Bringé, "L'impact des trajectoires familiales sur les parcours résidentiel et géographique des franciliens nés entre 1930 et $1950 »$, Revue européenne des sciences sociales [En ligne], 54-1 | 2016, mis en ligne le 15 mai 2019, consulté le 04 janvier 2020. URL : http:// journals.openedition.org/ress/3452 ; DOI : 10.4000/ress.3452 


\title{
L'IMPACT DES TRAJECTOIRES FAMILIALES SUR LES PARCOURS RÉSIDENTIEL ET GÉOGRAPHIQUE DES FRANCILIENS NÉS ENTRE 1930 ET 1950
}

\author{
CATHERINE BONVALET \& ARNAUD BRINGÉ \\ INED, Paris / INED, Paris \\ bonvalet@ined.fr / bringe@ined.fr
}

\begin{abstract}
Résumé. Au cours des cinquante dernières années, la France a connu de profonds changements urbains avec une diffusion sans précédent de la propriété qui a accompagné le phénomène de périurbanisation. II est possible de suivre, à travers l'enquête «Biographies et entourage», comment les générations nées entre 1930 et 1950 ont vécu ces changements urbains de leur enfance à nos jours et comment les comportements matrimoniaux ont affecté leurs trajectoires résidentielle et géographique. La sécurité que procure la propriété a de fortes chances de durer tant que les couples résistent à l'épreuve du temps. En revanche, elle apparait très compromise lorsque les conjoints se séparent. Quelles que soient les générations, les séparations et les divorces ont entrainé des bifurcations dans les «parcours logement» souvent brutales en interrompant des trajectoires ascendantes en termes de position résidentielle où le statut de propriétaire est considéré comme un signe de réussite sociale.
\end{abstract}

Mots-clés: parcours résidentiel, propriété, trajectoires familiales, trajectoires géographiques.
Abstract. France has undergone major urban transformations over the last fifty years, involving a process of peri-urbanization accompanied by an unprecedented growth in home ownership. Using data from the "Biographies et entourage" survey, it is possible to explore how the cohorts born between 1930 and 1950 have been affected by these urban changes, from their childhood up to the present day, and how their residential and geographical trajectories are influenced by marital beha- viors. The security associated with home ownership is likely to be durable, provided that the couples remain intact over time. It is compromised, however, if the partners separate. Across all cohorts, separation and divorce lead to an often brutal bifur- cation in residential trajectories that halts upward residential mobility, the status of homeowner being viewed as a sign of social success.

Keywords: family trajectories, geographical trajectories, ownership, residential trajectories. 


\section{INTRODUCTION}

Au cours des cinquante dernières années, la France a connu de profonds changements urbains avec une diffusion sans précédent de la propriété qui a accompagné le phénomène de périurbanisation, mais aussi la réhabilitation des centres villes. Grâce au développement des transports, mais également au développement du crédit qui a bouleversé le calendrier d'accès à la propriété, un nouveau mode de vie urbain à la périphérie des villes s'est peu à peu mis en place, fondé sur la séparation entre domicile et travail. Après la construction des grands ensembles des années 1960, on assiste à une véritable explosion pavillonnaire avec la création de «nouveaux villages» et de lotissements à la périphérie des communes rurales aux marges des agglomérations. L'acquisition d'une maison intervient très tôt, au moment même où se constitue la famille. «La question de l'achat d'une maison se pose avec une force particulière à certaines étapes du cycle de vie, en relation avec le souci de fonder une famille, c'est-à-dire au moment du mariage ou dans les années qui suivent, en liaison avec l'apparition des enfants » (Bourdieu, 2000, p. 50). C'est ainsi que, petit à petit - les politiques du logement ayant accompagné voire encouragé ce désir de propriété des ménages -, une trajectoire résidentielle type où la maison individuelle en accession à la propriété vient couronner une trajectoire résidentielle ascendante a correspondu à la trajectoire familiale type fondée sur la famille nucléaire (Bonvalet et Bringé, 20I3). Le renversement des tendances socio-démographiques de la fin des années I960 ayant remis en question l'idée même de modèle familial unique et de parcours familial linéaire, il a contribué à engendrer une plus grande mobilité résidentielle et géographique venant alors troubler le bel agencement des étapes du «parcours logement» qui va de la location à la propriété.

Cet article se propose de retracer les différents parcours des générations habitant Paris et la banlieue nées entre 1930 et 1950, à partir de l'enquête «Biographies et entourage » de l'Ined ${ }^{1}$. Ces générations ont la particularité d’avoir connu les grands changements urbains qui se sont produits en France 
avec le renouvellement du parc immobilier : amélioration du confort, diffusion de la propriété, périurbanisation (Bonvalet, 2007). Leur histoire présente la particularité de se situer à la charnière entre deux mondes: le monde rural du début du $\mathrm{xx}^{\mathrm{e}}$ siècle qu'ils ont connu enfants et le monde global du $\mathrm{XxI}^{\mathrm{e}}$ siècle auquel ils ont contribué. Par ailleurs, ces transformations urbaines ont accompagné les bouleversements qui ont traversé la société, aussi bien dans le monde du travail avec la participation massive des femmes que dans la sphère privée avec la multiplication et la diversité des étapes familiales, avec ou sans enfant. Il est donc possible de suivre à travers l'enquête «Biographies et entourage » comment des générations ont vécu ces changements urbains de leur enfance à nos jours et comment les comportements matrimoniaux ont affecté leurs trajectoires résidentielle et géographique. En effet, l'échantillon collecté (2 830 personnes) offre la possibilité d’analyser les différentes évolutions - familiale et résidentielle - et de les comparer selon les générations. Et, plus particulièrement, il permet d'examiner en quoi les premières générations du baby-boom (nées entre 1946 et 1950) qui ont participé aux transformations de la famille se distinguent, dans leur comportement résidentiel, des générations nées avant ou pendant la guerre.

Après avoir retracé les trajectoires géographiques et résidentielles des Franciliens âgés de 50 à 70 ans, ainsi que le contexte dans lesquelles elles se sont déroulées, nous essayerons d’analyser comment ces parcours sont liés aux trajectoires familiales, pour étudier ensuite l'impact d’un événement particulier, comme la naissance ou le divorce, sur la trajectoire résidentielle et géographique.

2830 enquêtés âgés de 50 à 70 ans et résidant en Île-de-France au moment de l'enquête. Elle recense notamment tous les logements habités plus d'un an par l'enquêté ainsi que leur localisation. Nous pouvons ainsi suivre toutes les migrations effectuées au cours du cycle de vie et étudier comment la répartition de cette génération dans l'espace parisien s'est modifiée avec le temps. 


\section{LES PARCOURS GÉOGRAPHIQUES ET RÉSIDENTIELS Des mouvements centrifuges liés à la diffusion de la propriété}

Les cinquante dernières années ont vu la région Île-de-France se transformer profondément, sous l'effet de plusieurs phénomènes : la rénovation de Paris, la densification des banlieues et la disparition des villages ruraux en grande couronne. Ces bouleversements ont modifié la répartition de la population. Entre 1962 et 2007, la population de l'Île-de-France s'est accrue de $40 \%$, légèrement plus rapidement que celle de la population de la France métropolitaine. La répartition des habitants à l'intérieur de la région Île-de-France s'est, quant à elle, nettement modifiée : d'environ un tiers en 1962, le poids de Paris est inférieur à un cinquième en 2007, ce qui correspond à une baisse de $43 \%$ (Imbert, 2012). Dans les années 1970, après la construction des grands ensembles de la décennie précédente, l'habitat pavillonnaire explose à la périphérie des communes rurales aux marges de l'agglomération parisienne, à travers un phénomène d'étalement urbain, qui se poursuit au cours de la période récente.

Graphique i. Évolution de la population des couronnes d'urbanisation de l'Île-de-France de I946 à 1999

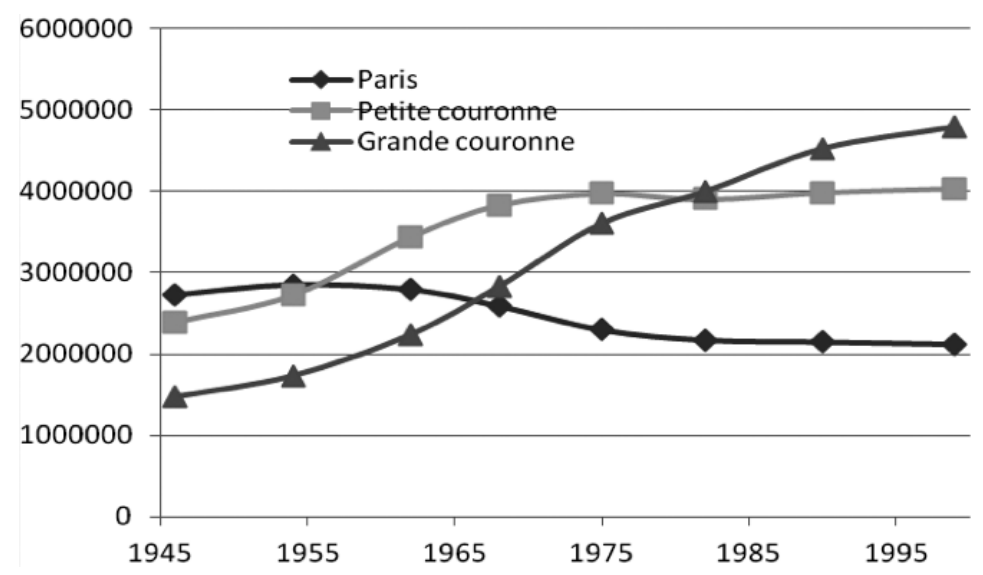

Source: Insee, recensements. 


\section{I.I. TRAJECTOIRES GÉOGRAPHIQUES \\ Un mouvement centrifuge dominant}

Les ménages franciliens appartenant aux générations 1930-1950 ont été des acteurs essentiels du renversement de modèle urbain (Berger, 2004). En s'installant en petite et grande couronne au fur et à mesure de la constitution de la famille, ils ont ainsi contribué à une nouvelle répartition de la population dans l'espace francilien, comme le montre leur changement de localisation dans le graphique 2 qui représente la répartition des localisations par âge. Comme point de départ, nous avons retenu l'âge de 20 ans, et de 50 ans comme point d'arrivée, âge où tous les enquêtés sont présents dans l'enquête ${ }^{2}$. Le poids de l'étranger et de la province s'estompent peu à peu avec les migrations au profit d'une localisation dans la petite couronne, puis dans la grande couronne.

Si l'on considère maintenant uniquement l'évolution de la localisation des individus présents à l'intérieur de la région Île-de-France à leurs 25 ans, les changements de la répartition spatiale de la population apparaissent clairement sous l'effet de deux mouvements concomitants avec, d'une part, une modification de poids relatif puisqu'une partie des provinciaux et étrangers s'installent directement en banlieue, et d'autre part, un mouvement centrifuge où Paris au fil des années perd une partie de ses habitants de cette génération. Si à 25 ans, $32 \%$ des enquêtés installés en Île-de-France se trouvent dans la capitale, ils ne sont plus guère que $20 \%$ à 50 ans. La proche couronne garde un poids à peu près stable au cours du cycle de vie des enquêtés : $39 \%$ à 25 ans, $37 \%$ à 50 ans. La grande couronne est la seule zone géographique dont le poids dans la répartition spatiale augmente au cours du cycle de vie : de $29 \%$ des effectifs à 25 ans à $43 \%$ à 50 ans.

2 L'enquête ne permet pas de saisir la trajectoire des Franciliens nés entre 1930 et 1950 qui ont quitté définitivement la région avant l'âge de 50 ans. 
Graphique 2. Évolution de la localisation géographique des enquêtés de 25 à 50 ans
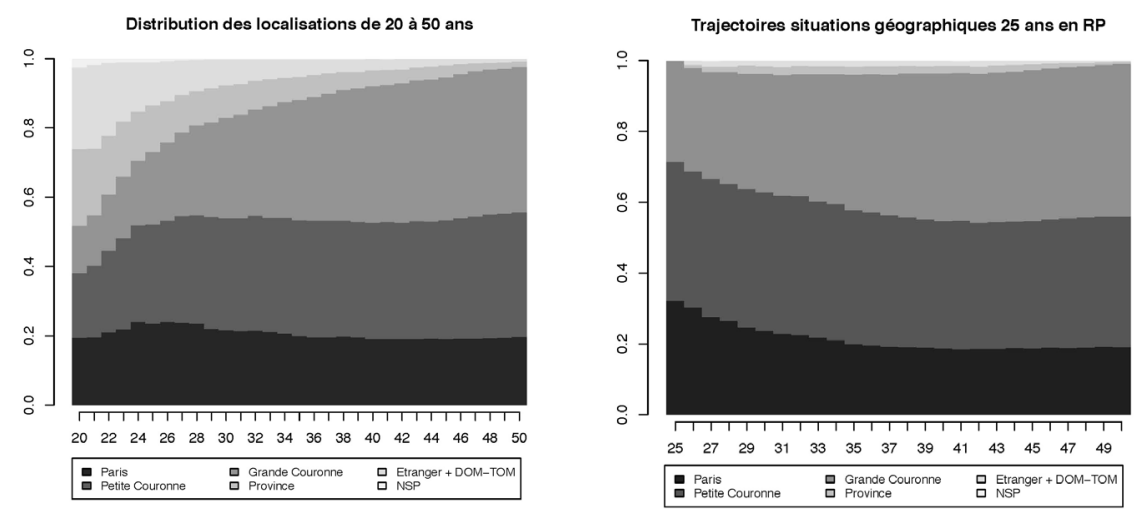

Source: Enquête «Biographies et entourage», INED, 2001.

Ces évolutions reflètent les changements urbains de l'île-de-France. En effet, Paris a commencé à se dépeupler dès 192I (Bonvalet et Tugault, 1984), la tendance s'accentuant nettement à partir de 1954. Aussi est-ce encore le cas dans les années 1960, lorsque la plupart des enquêtés ont quitté le foyer parental. En revanche, la population augmente fortement dans la petite couronne sous l'effet de la construction des grands ensembles ${ }^{3}$. La décennie suivante, cette politique est remise en cause par la reprise des constructions de maisons individuelles. L’agglomération parisienne s'étend avec l'émergence d’un phénomène de périurbanisation, liée au développement des liaisons ferroviaires et à l’accession à la propriété encouragée par les pouvoirs publics. Les générations enquêtées font partie des pionniers de l’accession à la propriété en périurbain dont le mode de vie diffère à la fois de celui du monde urbain et de celui du monde rural.

3 Cela explique les écarts constatés avec l'enquête «Peuplement et dépeuplement de Paris», menée en 1986 auprès de générations nées entre 1926 et 1935 (Bonvalet et Maison, 1996). D'après cette enquête, au milieu des années 1950, c'est-à-dire lorsque la majorité des enquêtés étaient déjà installés, Paris rassemblait encore $42 \%$ des individus résidant en Île-deFrance. Cette proportion n'est plus que de $32 \%$ une décennie plus tard d'après l'enquête «Biographies et entourage», ce qui traduit le début de la baisse de la population parisienne. 
Le graphique 3 qui illustre les trajectoires géographiques les plus représentées au sein de notre échantillon ${ }^{4}$, couvrant plus de la moitié des parcours des enquêtés (I 545), témoigne du processus de périurbanisation. On y voit l'importance des trajectoires qui se sont effectuées dans la même zone géographique. En effet, les trois types de trajectoires les plus fréquents se sont entièrement déroulés en petite couronne $(n=327)$, en grande couronne $(n=324)$ ou à Paris $(n=198)$. Viennent ensuite les mobilités au sein de la région Île-deFrance et les migrations des provinciaux vers l'agglomération parisienne.

Graphique 3. Trajectoires géographiques les plus fréquentes

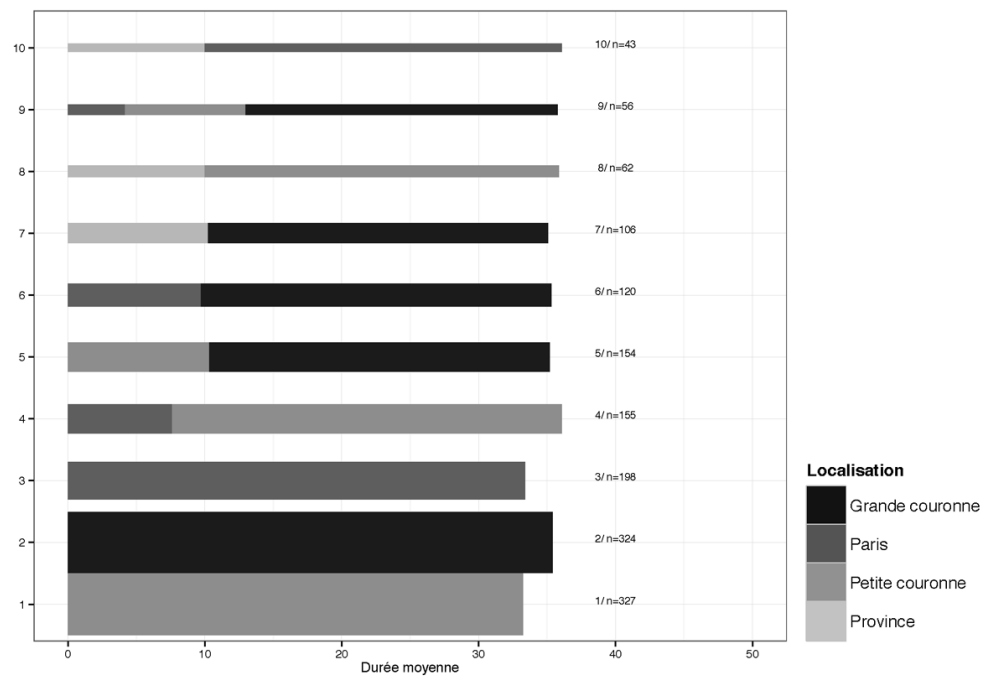

Source: Enquête «Biographies et entourage».

Champ: les I 545 enquêtés ayant l'une des trajectoires les plus fréquentes.

Note: La longueur des barres (axe des abscisses) correspond à la durée moyenne des trajectoires de chaque type. La proche banlieue correspond à la petite couronne et la grande banlieue à la grande couronne.

Lecture: 327 enquêtés ont une trajectoire géographique située intégralement en petite couronne, 155 ont commencé leur trajectoire à Paris avant de déménager en petite couronne, etc.

4 Un changement de résidence au sein d'une aire géographique n'est pas comptabilisé, seul l'est celui qui s'accompagne d'un changement d'aire. 
Les résultats précédents ne rendent compte que de la moitié des trajectoires suivies par les Franciliens âgés de 50 à 70 ans, l'autre moitié est le fait d'individus ayant eu des parcours plus complexes avec de nombreux allers et retours que ce soit à l'intérieur de la région ou hors de la région Île-de-France. En fait, si l'on considère maintenant l'ensemble des enquêtés mais en ne retenant au plus que les trois derniers changements d’aires géographiques (Graphique 4), les mobilités intra-urbaines en Île-de-France se caractérisent depuis plusieurs années par différents mouvements contradictoires. Le mouvement le plus important est centrifuge : 9 Io enquêtés (soit $32 \%$ de l'ensemble des enquêtés) se sont éloignés du centre de l'agglomération au cours de leur dernière étape, 587 (2I \%) pour s'installer en Grande Couronne - parmi eux, 20 I Parisiens (7\%) qui sont partis directement en grande couronne - et 386 qui ont quitté la petite couronne pour la périphérie. Par ailleurs, 323 Parisiens (I \%) ont quitté la capitale pour s'implanter en petite couronne.

Le deuxième mouvement géographique correspond à l'enracinement dans la même zone géographique : 857 enquêtés (30\%) sont restés stables d'un point du vue spatial: 327 (I2\%) en petite couronne, 324 (I \% ) en grande couronne, 198 (7\%) à Paris, et 8 personnes n'ont jamais décohabité. Quant au retour au centre de l'agglomération, il concerne 302 enquêtés, soit I I \% de l'ensemble de Franciliens âgés de 50 à 70 ans : I 19 enquêtés (4\%) ont quitté la grande banlieue pour habiter la petite banlieue et I83 individus (6\%) ont effectué un retour à Paris. Si on ajoute les I24 ménages franciliens qui après une étape résidentielle en province ou à l'étranger s'installent directement à Paris, on obtient le pourcentage de $10 \%$ de retour à Paris. Toutes les autres trajectoires (559 enquêtés; soit $20 \%$ ) comportent des allers et retours entre la région île-de-France et la province ou l'étranger.

Le tableau i montre que contrairement au schéma classique qui ferait passer l'ensemble des ménages par Paris, seulement près de la moitié des enquêtés ont vécu un moment dans la capitale: de grandes différences peuvent être observées. Si la majorité des Parisiens de naissance et les migrants provinciaux et étrangers ont séjourné à Paris, les habitants de la banlieue au contraire sont beaucoup moins nombreux à avoir habité Paris, adultes : $38 \%$ des enquêtés nés en Petite 
Couronne et $24 \%$ en Grande Couronne. Cela confirme les mouvements centrifuges et les mobilités à l'intérieur d'une même zone géographique hors Paris.

Graphique 4. Mobilités intra-urbaines en Île-de-France
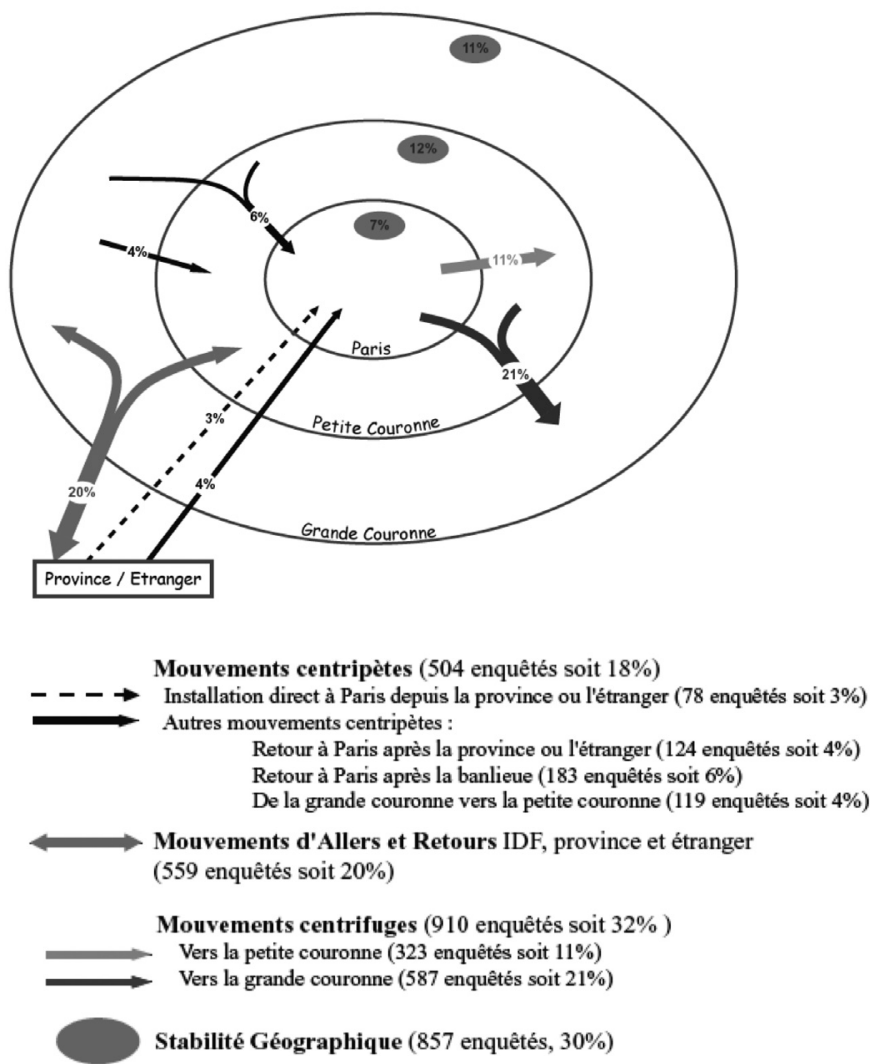

Source: Enquête «Biographies et entourage».

Champ: 2830 enquêtés. 
Tableau I. Proportion d'enquêtés à avoir vécu dans les différentes aires géographiques au cours de la vie adulte, selon leur origine

\begin{tabular}{|l|c|c|c|c|c|c|}
\hline & PARIS & $\begin{array}{c}\text { PETITE } \\
\text { COURONNE }\end{array}$ & $\begin{array}{c}\text { GRANDE } \\
\text { COURONNE }\end{array}$ & PROVINCE & ÉTRANGER & EFFECTIF \\
\hline NÉS À PARIS & $56 \%$ & $65 \%$ & $45 \%$ & $17 \%$ & $11 \%$ & 541 \\
\hline $\begin{array}{l}\text { NÉS EN PETITE } \\
\text { COURONNE }\end{array}$ & $38 \%$ & $71 \%$ & $44 \%$ & $13 \%$ & $7 \%$ & 315 \\
\hline $\begin{array}{l}\text { NÉS EN GRANDE } \\
\text { COURONNE }\end{array}$ & $24 \%$ & $29 \%$ & $84 \%$ & $12 \%$ & $8 \%$ & 266 \\
\hline $\begin{array}{l}\text { NÉS EN PROVINCE } \\
\text { NÉS À L'ÉTRANGER } \\
\text { OU DANS LES } \\
\text { DOM-TOM }\end{array}$ & $53 \%$ & $57 \%$ & $56 \%$ & $49 \%$ & $19 \%$ & 1065 \\
\hline ENSEMBLE & $49 \%$ & $51 \%$ & $47 \%$ & $24 \%$ & $43 \%$ & 643 \\
\hline
\end{tabular}

Source: Enquête «Biographies et entourage».

Champ: 2830 enquêtés.

Lecture: Par exemple, $65 \%$ des enquêtés nés à Paris ont vécu au moins un an en petite couronne après avoir quitté le domicile parental.

Ces résultats traduisent les mobilités que les générations nées entre 1930 et 1950 ont connues au cours des quarante dernières années. Ils confirment le mouvement centrifuge, avec notamment le processus de périurbanisation, mais révèlent également un phénomène de stabilité à l'intérieur d’une même zone géographique. Quant au retour au centre, il reste très minoritaire.

\section{I.2. TRAJECTOIRES RÉSIDENTIELLES}

Une forte diffusion de la propriété avec une complexité de plus en plus grande des parcours

L'enquête «Biographies et entourage » montre que les enquêtés ont effectué leurs parcours résidentiel dans un contexte de diffusion de la propriété et d'urbanisation sans précédent ${ }^{5}$. Ce qui était une utopie au XIX ${ }^{\mathrm{e}}$ siècle et un demi-échec dans l'entre deux guerres se concrétise enfin au cours de la seconde moitié du Xx siècle (Bonvalet, 1998). En 1954, 34\% seulement des

5 À 10 ans, 38\% des enquêtés qui vivaient dans leur famille avaient des parents ou grands parents propriétaires du logement. 
ménages étaient propriétaires contre $56 \%$ en 2002. Mais cette diffusion de la propriété ne s'est pas faite de manière linéaire (Topalov, 1987) et porte l'empreinte des grandes étapes de la politique du logement. Les années 1950-1963, qui correspondent à la période où les enquêtés plus âgés deviennent indépendants et constituent une famille, se caractérisent par une forte croissance de la production de logements neufs, notamment de logements destinés à la propriété. Cette période est suivie, de 1963 à 1968, d’un fléchissement de l'accession à la propriété puis d'une reprise mais à un rythme moins rapide que celui des années 1950. Après 1974, bien que la construction de maisons individuelles en accession à la propriété, favorisée par la réforme des aides au logement de 1977, se maintienne, et s'accroisse même au cours des années I980, on note de nouveau une baisse du taux d'accession à la propriété. Les enquêtés selon les générations vont donc bénéficier de conditions plus ou moins favorables. Les cohortes nées juste avant la guerre ont profité du contexte très favorable des années 1955-1965 pour devenir rapidement propriétaires $^{6}$. En raison du retournement de la conjoncture économique et du ralentissement de l'inflation après 1980, les plus jeunes connaîtront un environnement économique un peu moins propice, mais la réforme du logement de 1977, avec la mise en place des prêts aidés à la propriété (PAP) et des aides au logement (APL) ${ }^{7}$, compensera largement ces effets négatifs.

Ces différences d'accès à la propriété de la résidence principale entre générations se retrouvent illustrées dans le graphique 5. À 35 ans, $48 \%$ des enquêtés nés entre 1935 et 1939 sont déjà propriétaires contre $40 \%$ de ceux de la génération du baby-boom. Ces différences subsistent puisque, à 50 ans, le taux de propriétaires chez les plus âgés s'élève à plus de $66 \%$ contre $58 \%$ chez les plus

6 En effet, les générations nées entre les deux guerres sont celles qui vont profiter de toutes les transformations des modes de financement du logement. À partir des années 1950, le crédit se développe avec les prêts du Crédit foncier et les ménages qui au départ étaient réticents à l'idée même de crédit se laissent d'autant plus séduire par l'accession à la propriété que la loi de 1948 met fin au blocage des loyers (Loiseau et Bonvalet, 2005).

7 Les Franciliens appartenant à ces générations ont été moins propriétaires que les générations qui les précèdent mais, à l'échelle de la France entière, les baby-boomers ont des taux équivalents aux générations nées dans les années 1930. 
jeunes (1946-1950). Mais, contrairement aux générations 1930-1934, les plus jeunes ont pu bénéficier dès le début de leur trajectoire résidentielle des nouveaux logements HLM construits à partir de la fin des années 1950 (à 25 ans, on trouve I०\% parmi les générations du baby-boom contre $3 \%$ des générations 1930-1934).

Ces résultats offrent une vision assez simple : à 50 ans les ménages enquêtés ont, depuis leur premier logement indépendant, occupé en moyenne un nombre de logements proche de trois et $62 \%$ sont propriétaires de leur logement. L’analyse des trajectoires résidentielles révèle cependant une réalité plus complexe. En retenant 6 statuts possibles : propriétaires, locataires privés, locataires HLM, autres statuts (logements gratuits, logements de fonction, logement dans la famille, hôtels, etc.), logés dans une collectivité, logements transitoires $^{8}$, on obtient 53I trajectoires possibles. Si le nombre d'étapes, c'est-à-dire le nombre de changements de statuts (et non pas de logements) varie de I à I4, il existe une très forte concentration autour de 2 à 3 étapes (Bonvalet, 2007).

Au-delà de 6 étapes, les trajectoires sont pratiquement le fait de quelques individus, voire d'un individu. C'est pourquoi, parmi les 53 I trajectoires, nous n’avons retenu que les io trajectoires les plus fréquentes. Ces dernières, représentées dans le graphique 6, correspondent aux parcours de la moitié environ des enquêtés de «Biographies et entourage ». La trajectoire souvent présentée comme typique (ou «trajectoire modale», Grafmeyer, 1994) avec le passage du statut de locataire à celui de propriétaire concerne seulement $20 \%$ des enquêtés et $25 \%$ si l'on ajoute ceux qui ont commencé par une étape comme logés par la famille ou chez l'employeur. Viennent ensuite les enquêtés qui ont, quant à eux, un seul logement, que ce soit en location privée $(7 \%)$ ou en propriété (7\% également).

8 Ont été regroupées sous cette rubrique les périodes de plus d'un an durant lesquelles les enquêtés ont occupé plusieurs logements moins d'un an. Par exemple, 3 chambres meublées durant 6 mois, 9 mois et 3 mois seront comptabilisées comme une seule période transitoire. 
Graphique 5. Trajectoires résidentielles par générations
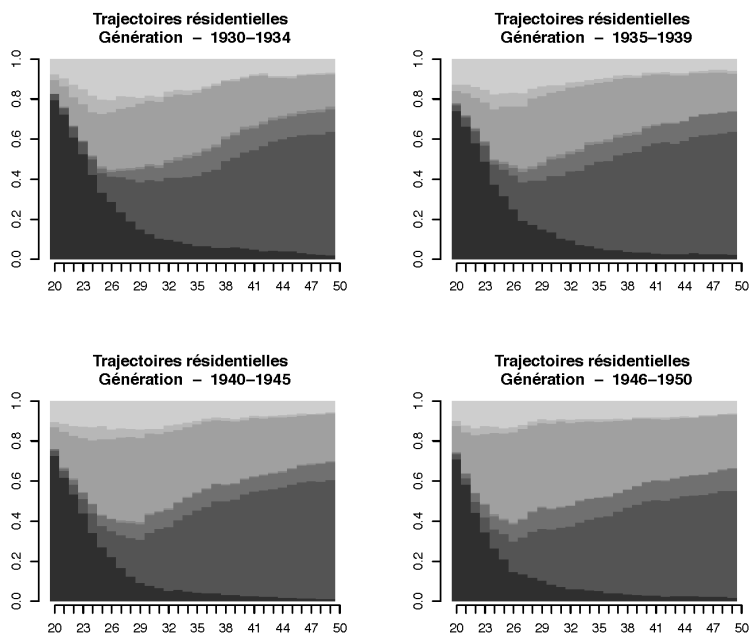

\begin{tabular}{|llll|}
\hline$\square$ Non indépendant & $\square$ Loi de 48 & $\square$ Autres statuts \\
$\square$ Propriétaire & $\square$ Locatif privé & \\
$\square$ HLM & $\square$ Autres locataires & \\
\hline
\end{tabular}

Graphique 6. Trajectoires résidentielles les plus fréquentes

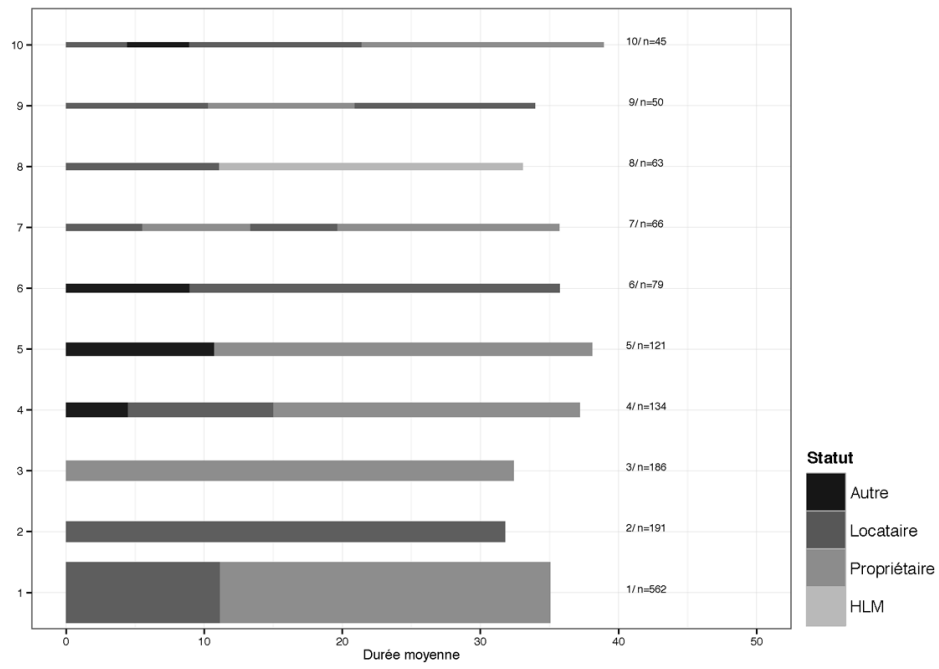

Source: 50 \% de l'échantillon de l'enquête «Biographies et entourage». 
Les trajectoires résidentielles et géographiques des générations nées entre I930 et 1950 témoignent des grands moments de l'urbanisation francilienne qui se sont opérés au cours de la deuxième moitié du $\mathrm{xx}^{\mathrm{e}}$ siècle avec, notamment, la diffusion de la propriété, le phénomène de la périurbanisation et de la gentrification. Du point de vue géographique, trois grandes tendances se dégagent: un mouvement centrifuge qui éloigne les Franciliens du centre de l'agglomération, une certaine stabilité dans la zone géographique où l'on s'est implanté en Île-de-France, dénotant un attachement à Paris ou un enracinement en banlieue, et, dans une moindre proportion, un retour au centre de l'agglomération. À l'intérieur de chaque zone géographique et a fortiori à l'intérieur de la région Île-de-France, les enquêtés ont effectué des choix tant du point de vue du statut d'occupation que de la localisation en fonction de l'évolution de leur position dans l'échelle sociale au cours de leur vie : ils ont cherché à faire correspondre leur position résidentielle à leur position sociale en allant habiter ou en demeurant dans des communes qui regroupent leurs pairs, quitte à rester locataires pour vivre à Paris. Certains ont initié des mouvements urbains, comme par exemple des pionniers de la périurbanisation avec l'accession à la propriété d'une maison individuelle en lotissement ou encore des pionniers de la gentrification dans certains arrondissements de la capitale ou en banlieue proche. Dans ce cas, ces mobilités ont été permises par les acteurs locaux et nationaux qui ont alors adapté l'offre de logements et d'infrastructures (transports, écoles, etc.). Dans d’autres cas, les mobilités ont été le résultat d'une politique favorisant la propriété qui a entraîné une déconcentration urbaine, les ménages s'éloignant du centre ville pour acquérir leur logement.

\section{PARCOURS RÉSIDENTIELS ET TRAJECTOIRES FAMILIALES}

L’analyse par génération montre bien l'effet différencié des politiques du logement sur les parcours résidentiels et géographiques. Toutefois, on sait que les mutations de la famille dont les baby-boomers ont été les principaux acteurs (Segalen, 20ıо ; Bonvalet et al., 20I I) n’ont pas été sans conséquence sur l'évolution globale de la propriété (Bonvalet et Bringé, 20I3). Comment les trajectoires familiales des différentes générations (1930-1934, 1935-1939, 1940-1945, 1946-1950) 
portent-elles la trace des évolutions du couple et de la famille? Comment ont-elles impacté les trajectoires géographiques et résidentielles?

\section{I. DIVERSITÉ DES TRAJECTOIRES FAMILIALES}

À partir de l'enquête «Situations familiales» de l'INED de 1985 , Laurent Toulemon (1994) a mis en évidence la succession d'étapes qui ont transformé les parcours familiaux, chacune des générations évoluant sur des territoires inconnus et inventant chaque fois de nouveaux modèles : travail salarié, cohabitation juvénile, cohabitation adulte et, un peu plus tard, divorce. Par bien des aspects, les générations nées après-guerre sont des générations de transition, charnières, initiant des comportements plus autonomes à l'égard du couple et de la famille et réinvestissant des formes familiales et conjugales (comme le concubinage, les familles recomposées) vécues alors sous l’angle de la libération (Bonvalet et al, 20 I).

On retrouve dans le graphique 7 cette succession d'étapes, la cohabitation juvénile n’apparaît pratiquement pas dans les générations les plus vieilles ( $0,7 \%$ chez les hommes de 20 ans et I \% chez les femmes) et se diffuse progressivement de génération en génération: parmi les premiers baby-boomers, ces pourcentages atteignent respectivement $2,6 \%$ et 3,4\%. À 25 ans, la proportion de cohabitants est trois fois plus forte parmi les femmes nées après guerre que parmi les femmes nées au début des années 1930. Il en est de même de la cohabitation hors mariage ou de la vie solitaire après une séparation. La diffusion de la cohabitation est spectaculaire puisque, à 35 ans, moins de I \% des hommes appartenant aux générations 1930-1934 vivent en couple sans être mariés après une rupture conjugale, ils sont près de $6 \%$ chez les baby-boomers au même âge. 
Graphique 7. Trajectoires familiales des hommes et des femmes selon les générations
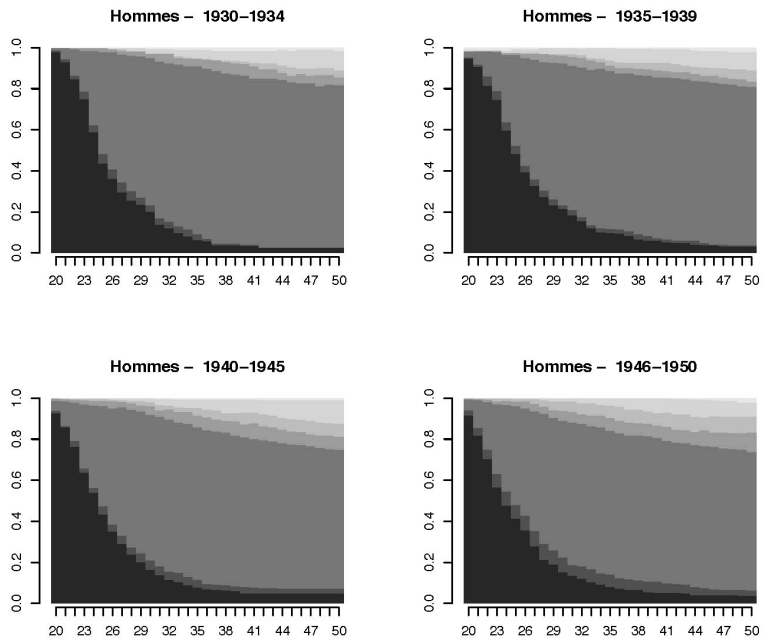

$\begin{array}{lllllllllll}20 & 23 & 26 & 29 & 32 & 35 & 38 & 41 & 44 & 47 & 50\end{array}$
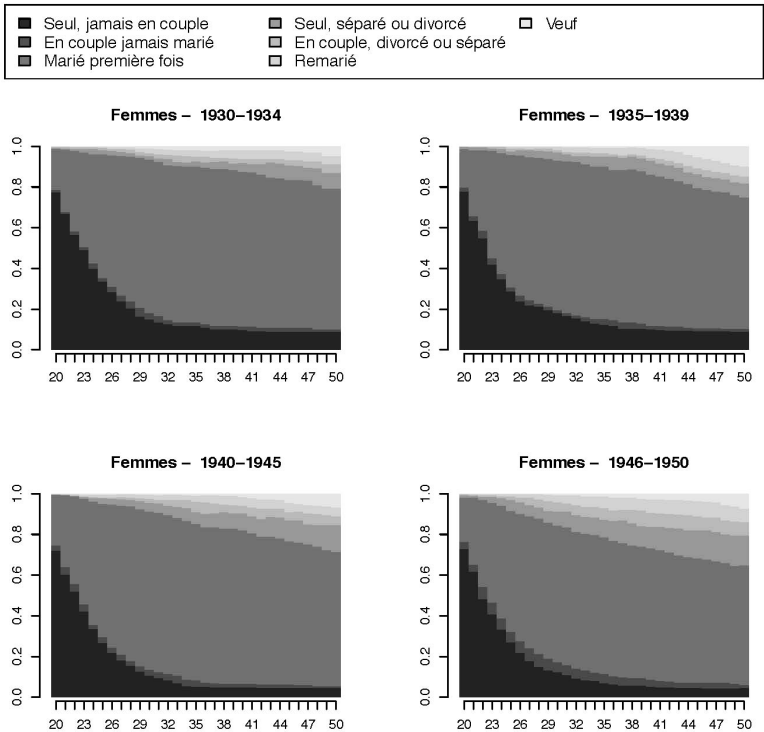

Seul, jamais en couple En couple jamais marié Marié première fois
ㅁ Seul, séparé ou divorcé
En couple, divorcé ou séparé Remarié

$\square$ Veuf

Source: Enquête «Biographies et entourage», INED, 200 I. 
Quant aux femmes, les transformations les plus importantes proviennent davantage de l'augmentation et de la durée de la vie solitaire après une séparation: à 35 ans, le pourcentage de femmes seules est multiplié par 3 alors que celui des femmes en couple non marié est multiplié par 2.

Conséquence de tous ces bouleversements, la baisse de la part des personnes toujours mariés pour la première fois à 50 ans. Ils représentaient $79 \%$ des hommes et $69 \%$ des femmes des générations les plus vieilles contre les deux tiers des hommes et la moitié des femmes des générations nées après-guerre.

Ainsi, que ce soient par la cohabitation hors mariage, les séparations ou les divorces, les enfants du baby-boom ont-ils réussi à faire admettre par l'ensemble de la société la fin d’un certain nombre de règles morales qui régissaient la famille, et à faire accepter - non sans peine - d'autres façons de vivre leur couple ainsi que d'autres manières d'être parents en dehors de la cellule nucléaire. Ils ont autorisé, réactualisé, modernisé, d’autres formes de vie familiale en banalisant la cohabitation hors mariage, en dédramatisant les naissances «illégitimes», les séparations, les divorces, tout en servant de modèles aux générations précédentes dans la mesure où ils leur ont permis à leur tour d'échapper à la morale familiale des années 1950-1960 (Bonvalet et al., 20II). Cette mutation a engendré de nouvelles trajectoires familiales, moins linéaires, où peuvent alterner périodes de vie solitaire et périodes de vie en couple, avec ou sans enfant.

\subsection{TRAJECTOIRES FAMILIALES ET RÉSIDENTIELLES Des parcours liés}

L'émergence d'un nouveau parcours familial moins linéaire va se traduire par une mobilité résidentielle accrue et surtout remettre en question les politiques du logement uniquement fondées sur l'accession à la propriété qui reposait essentiellement sur la stabilité du couple (Bonvalet et Merlin, 1988). En effet, depuis la fin du XVIII ${ }^{\mathrm{e}}$ siècle, l'histoire de la famille apparaissait très liée à celle de la propriété, le complexe famille/propriété fournissant les bases de tout un courant de pensée dont Bonald et Le Play sont les représentants. Pour les contre-révolutionnaires, l'indépendance de la famille est garantie par la propriété (Gengembre, 1989). Pour Jules Siegfried, famille et propriété donnent les joies les plus douces. La 
relation famille nucléaire / accession à la propriété apparaît bien comme l’avatar moderne du couple famille / propriété du XIX siècle. Christian Topalov considère en effet que «l'apparition de la propriété marchande et capitaliste du logement est contemporaine des ruptures sociales qui conditionnent la naissance de la famille nucléaire [...]. Le cycle de la marchandise logement repose sur l'usage et le paiement de celle-ci par une unité de type familiale» (Topalov, 1987, p. 16). Ainsi, l'accession à la propriété n’a-t-elle pu se développer qu'en symbiose avec le modèle de la famille nucléaire au fur et à mesure que ce dernier s'imposait dans les sociétés urbaines. Dès les années 1960, la maison en propriété apparaît comme le cadre idéal pour accueillir des enfants (Raymond et al., 1966 ; Bourdieu 1990). La réforme du logement de 1977 resserrera encore plus les liens historiques entre achat du logement et constitution de la famille. L'enquête «Biographies et entourage» montre que les enquêtés vont alors bénéficier des opportunités offertes par la construction de nombreux logements neufs, notamment des pavillons en périurbain ainsi que des aides à la propriété associées.

Ils accèderont à la propriété en moyenne 6 ans après s'être mariés, à l'âge de 32 ans environ, soit 4 ans après la naissance du premier enfant et $\mathrm{I}$ an seulement après l'arrivée du deuxième enfant. Compte tenu des différences d'âge entre conjoints, les femmes deviennent propriétaires plus jeunes que les hommes (32,5 ans contre 34 ans). Toutefois, sans nous étendre davantage, on notera que la stabilité résidentielle ne passe pas forcément par la propriété et que l'accès au HLM permet également aux couples de consolider leur statut sur le marché du logement en sortant du parc locatif privé et en se protégeant ainsi des hausses de loyer et d'une reprise éventuelle du logement par le propriétaire bailleur. Cette sécurité résidentielle que procurent l'achat ou l'obtention d'un logement social intervient quasiment au même âge entre 30 et 32 ans.

Cependant des différences entre générations apparaissent : les baby-boomers accèdent à la propriété un an plus jeune que les générations nées avant guerre, (pour les hommes 32,2 ans contre 33,5). En effet, atteignant la trentaine au moment même où intervient la réforme du logement en 1977, ils ont pu bénéficier des nouveaux prêts aidés à l'accession à la propriété (PAP) qui leur ont permis d'acheter plus rapidement leur logement. 
Tableau 2. Âge moyen aux différents événements familiaux et résidentiels

\begin{tabular}{|l|c|c|c|c|c|}
\hline & $\begin{array}{c}\text { ÂGE MOYEN } \\
\text { IER MARIAGE }\end{array}$ & $\begin{array}{c}\text { ÂGE MOYEN } \\
\text { IER ENFANT }\end{array}$ & $\begin{array}{c}\text { AGE MOYEN } \\
\text { 2E ENFANT }\end{array}$ & $\begin{array}{c}\text { ÂGE MOYEN } \\
\text { IER ACHAT }\end{array}$ & $\begin{array}{c}\text { ÂGE MOYEN } \\
\text { IER HLM }\end{array}$ \\
\hline MARIÉS & 25.1 & 27.9 & 30.6 & 31.8 & 30.2 \\
\hline HOMMES & 26.4 & 30.0 & 38.8 & 32.6 & 31.3 \\
\hline FEMMES & 23.7 & 25.8 & 28.8 & 31.0 & 28.9 \\
\hline $\begin{array}{l}\text { GÉNÉRATION } \\
\text { I930-1939 }\end{array}$ & 25.3 & 27.9 & 30.7 & 32.2 & 31.9 \\
\hline $\begin{array}{l}\text { GÉNÉRATION } \\
\text { I940-1945 }\end{array}$ & 24.7 & 27.8 & 30.7 & 31.8 & 30.6 \\
\hline $\begin{array}{l}\text { GÉNÉRATION } \\
\text { I946-1950 }\end{array}$ & 25.2 & 28.1 & 30.6 & 31.4 & 28.6 \\
\hline
\end{tabular}

Source: Enquête «Biographies et entourage», INED, 200 I.

Les liens entre trajectoires familiales et trajectoires résidentielles étant confirmées dans les analyses précédentes, il s'agit maintenant de mesurer, à l'aide de modèles de Cox, l'impact d'un événement démographique sur l'accès à la propriété. Les analyses effectuées montrent que l'effet du mariage suivi ou non d'enfants est très fort avec un risque instantané de devenir propriétaire multiplié par 3,3 à partir de la mise en couple officielle. Quant aux naissances, celle du premier enfant apparaît jouer un rôle légèrement moins important que celui du deuxième (risque instantané augmenté de 4I \% pour l'impact d'une deuxième naissance, de 3I \% pour l'impact d'une première naissance). Ces effets diffèrent selon les générations. Les naissances ne semblent pas avoir significativement pesé sur les trajectoires résidentielles des générations nées entre 1930 et 1934. En revanche, pour les premiers baby-boomers qui sont arrivés à l'âge où l'on constitue sa famille et où l'on recherche la stabilité résidentielle au moment de la réforme du logement de 1977, les effets du mariage et de la naissance des enfants sur le risque d’accéder à la propriété deviennent positifs. 

Catherine Bonvalet \& Arnaud Bringé: L'impact des trajectoires familiales...

Tableau 3. Modèle de Cox, impact sur l'accès à la propriété ensemble et par génération

\begin{tabular}{|c|c|c|c|c|}
\hline & & RÉGRESSION I & RÉGRESSION 2 & RÉGRESSION 3 \\
\hline \multirow{7}{*}{ DIPLÔME } & Aucun Diplôme & $0,522 * * * *$ & 0,543 ***** & 0,408 ****** \\
\hline & Inférieur au Bac & 0,862 *** & ns & ns \\
\hline & $\mathrm{Bac}$ & (I Réf.) & (I Réf.) & (I Réf.) \\
\hline & De Bac à Bac+2 & ns & ns & ns \\
\hline & Licence, Maitrise & $1,243 * *$ & $1,186 *$ & ns \\
\hline & Capes, Dea, Agrégation,... & I,276*** & $1,215^{* * *}$ & ns \\
\hline & Écoles Ingénieurs & 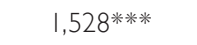 & I,429**** & ns \\
\hline \multirow{4}{*}{ GÉNÉRATION } & $1930-1934$ & (I Réf.) & (I Réf.) & (I Réf.) \\
\hline & $1935-1939$ & I,130* & ns & ns \\
\hline & $1940-1945$ & ns & ns & ns \\
\hline & $1946-1950$ & ns & ns & ns \\
\hline \multirow{4}{*}{ ORIGINE } & Paris & ns & ns & $0,844 *$ \\
\hline & Banlieue & ns & ns & ns \\
\hline & Province & (I Réf.) & (I Réf.) & (I Réf.) \\
\hline & Autres & $0,687 * * * * *$ & $0,688 * * * *$ & $0,625 * * * *$ \\
\hline \multicolumn{2}{|l|}{ IER MARIAGE } & $3,298 * * * *$ & & \\
\hline \multicolumn{2}{|l|}{ I RE NAISSANCE } & & $1,312 * * * *$ & \\
\hline \multicolumn{2}{|l|}{$2^{\mathrm{E}}$ NAISSANCE } & & & $|, 4| 4$ ***** \\
\hline
\end{tabular}

Génération 1930-1934

\begin{tabular}{|c|c|c|c|c|}
\hline & & RÉGRESSION I & RÉGRESSION 2 & RÉGRESSION 3 \\
\hline \multirow{7}{*}{ DIPLÔME } & Aucun Diplôme & ns & Ns & $0.504 * *$ \\
\hline & Inférieur au Bac & ns & Ns & ns \\
\hline & $\mathrm{Bac}$ & (I Réf.) & (I Réf.) & (I Réf.) \\
\hline & $\mathrm{De} B a c$ à $\mathrm{Bac}+2$ & ns & Ns & ns \\
\hline & Licence, Maitrise & ns & Ns & $3.587 *$ \\
\hline & Capes, Dea, Agrégation,... & ns & Ns & ns \\
\hline & Écoles Ingénieurs & I.883** & 1.653* & ns \\
\hline
\end{tabular}




\begin{tabular}{|c|c|c|c|c|}
\hline \multirow{4}{*}{ ORIGINE } & Paris & ns & ns & ns \\
\hline & Banlieue & ns & ns & ns \\
\hline & Province & (I Réf.) & (I Réf.) & (I Réf.) \\
\hline & Autres & ns & ns & ns \\
\hline \multicolumn{2}{|c|}{ IER MARIAGE } & 2.861 米米米 & & \\
\hline \multicolumn{2}{|c|}{ IRE NAISSANCE } & & ns & \\
\hline \multicolumn{2}{|c|}{$2^{\mathrm{E}}$ NAISSANCE } & & & ns \\
\hline
\end{tabular}

Génération 1935-1939

\begin{tabular}{|c|c|c|c|c|}
\hline & & RÉGRESSION I & RÉGRESSION 2 & RÉGRESSION 3 \\
\hline \multirow{7}{*}{ DIPLÔME } & Aucun Diplôme & $0.597 * *$ & 0.621 *** & $0.349 * * * *$ \\
\hline & Inférieur au Bac & ns & ns & ns \\
\hline & $\mathrm{Bac}$ & (I Réf.) & (I Réf.) & (I Réf.) \\
\hline & $\mathrm{De} B a c$ à $\mathrm{Bac}+2$ & ns & ns & ns \\
\hline & Licence, Maitrise & 1.897*** & I.83|*** & ns \\
\hline & Capes, Dea, Agrégation,... & ns & ns & ns \\
\hline & Écoles Ingénieurs & ns & ns & ns \\
\hline \multirow{4}{*}{ ORIGINE } & Paris & ns & $0.787 *$ & $0.632 * *$ \\
\hline & Banlieue & ns & ns & ns \\
\hline & Province & (I Réf.) & (I Réf.) & (I Réf.) \\
\hline & Autres & $0.562 * * *$ & $0.564 * * * *$ & $0.398 * * *$ \\
\hline \multicolumn{2}{|c|}{ IER MARIAGE } & 4.157 ***** & & \\
\hline \multicolumn{2}{|c|}{ I RE NAISSANCE } & & 1.340**** & \\
\hline \multicolumn{2}{|c|}{$2^{\mathrm{E}}$ NAISSANCE } & & & |. $48 \mid$ 米米 \\
\hline
\end{tabular}

Génération 1940-1945

\begin{tabular}{|c|c|c|c|c|}
\hline & & RÉGRESSION I & RÉGRESSION 2 & RÉGRESSION 3 \\
\hline \multirow{7}{*}{ DIPLÔME } & Aucun Diplôme & $0.427 * * * *$ & $0.449 * * * *$ & $0.359 * * * *$ \\
\hline & Inférieur au Bac & $0.73 \mid$ *** & ns & ns \\
\hline & Bac & (I Réf.) & (I Réf.) & (I Réf.) \\
\hline & $\mathrm{De} B a c$ à $\mathrm{Bac}+2$ & ns & ns & ns \\
\hline & Licence, Maitrise & ns & ns & ns \\
\hline & Capes, Dea, Agrégation,... & ns & ns & ns \\
\hline & Écoles Ingénieurs & 1.697******* & 1.664****** & ns \\
\hline
\end{tabular}


Catherine Bonvalet \& Arnaud Bringé: L'impact des trajectoires familiales...

\begin{tabular}{|c|c|c|c|c|}
\hline \multirow{4}{*}{ ORIGINE } & Paris & ns & ns & ns \\
\hline & Banlieue & ns & ns & ns \\
\hline & Province & (I Réf.) & (I Réf.) & (I Réf.) \\
\hline & Autres & 0.747******* & $0.764 * *$ & ns \\
\hline \multicolumn{2}{|c|}{ IER MARIAGE $^{\text {ER }}$} & 3.164**** & & \\
\hline \multicolumn{2}{|c|}{ IRE NAISSANCE } & & I. $308 * * * *$ & \\
\hline \multicolumn{2}{|c|}{$2^{\mathrm{E}}$ NAISSANCE } & & & I.477米米 \\
\hline
\end{tabular}

Génération 1946-1949

\begin{tabular}{|c|c|c|c|c|}
\hline & & RÉGRESSION I & RÉGRESSION 2 & RÉGRESSION 3 \\
\hline \multirow{6}{*}{ DIPLÔME } & Aucun Diplôme & 0.452 ***** & $0.472 * * * *$ & $0.425 * * *$ \\
\hline & Inférieur au Bac & ns & ns & ns \\
\hline & $\mathrm{Bac}$ & (I Réf.) & (I Réf.) & (I Réf.) \\
\hline & $\mathrm{De} \mathrm{Bac}$ à $\mathrm{Bac}+2$ & ns & ns & ns \\
\hline & Licence, Maitrise & ns & ns & ns \\
\hline & Capes, Dea, Agrégation,... & I.549**** & I. $444 * *$ & I.488* \\
\hline \multirow{5}{*}{ ORIGINE } & Écoles Ingénieurs & $1.462 * *$ & ns & ns \\
\hline & Paris & ns & ns & ns \\
\hline & Banlieue & ns & ns & ns \\
\hline & Province & (I Réf.) & (I Réf.) & (I Réf.) \\
\hline & Autres & $0.697 * * * *$ & $0.679 * * * *$ & $0.547 * * * *$ \\
\hline \multicolumn{2}{|c|}{ IER MARIAGE } & $3.17 \mid$ ****** & & \\
\hline \multicolumn{2}{|c|}{ I RE NAISSANCE } & & 1.355**** & \\
\hline \multicolumn{2}{|c|}{$2^{\mathrm{E}}$ NAISSANCE } & & & 1.670**** \\
\hline
\end{tabular}

Source: Enquête «Biographies et entourage », INED, 200 l.

****ignificatif au seuil de $1 \%$;

** Significatif au seuil de 5\%;

*Significatif au seuil de 10\%.

La constitution de la famille va ainsi de pair avec l'acquisition d'un logement dont le remboursement s'effectue sur plusieurs années, voire dizaines d’années. Le projet familial et le projet résidentiel reposent d’abord sur la durée et constituent de plus en plus une sorte de pari sur la stabilité du couple. Pour la majorité des ménages, l'accession à la propriété est une affaire de couple où les 
deux membres contribuent selon leurs ressources et éventuellement selon les ressources de leurs familles respectives. Lorsque le couple se sépare, les cartes sont redistribuées en fonction des mises de départ dans l'achat du logement et des revenus de chaque conjoint. Ce qui créait du lien et du sens dans l'accession à la propriété se trouve remis en cause avec la rupture du couple, à savoir créer une maisonnée qui s’inscrit dans le temps long des générations.

Les séparations et les divorces viendront bousculer des «parcours logement» considérés comme des parcours ascendants, l’achat du logement étant le signe d'une position sociale et d'une réussite familiale. Parmi les 2579 enquêtés mariés, $19 \%$ connaîtront un divorce. Ce pourcentage augmente fortement au fil des générations passant de $13 \%$ pour les générations d'avant-guerre à $23 \%$ pour les baby-boomers. La séparation du couple intervient en moyenne au bout de 12 ans de mariage ${ }^{9}$ et coïncide souvent avec l'abandon du statut de propriétaire comme le suggère la proximité des âges moyens au divorce et à la sortie de la propriété.

Tableau 4. Calendrier des événements familiaux des hommes et femmes ayant connu un divorce

\begin{tabular}{|l|c|c|c|c|c|}
\hline & $\begin{array}{c}\text { ÂGE MOYEN } \\
\text { IER MARIAGE }\end{array}$ & $\begin{array}{c}\hat{A} G E \text { MOYEN } \\
\text { IER ACHAT }\end{array}$ & $\begin{array}{c}\text { ÂGE MOYEN } \\
\text { SÉPARATION }\end{array}$ & $\begin{array}{c}\text { ÂGE MOYEN } \\
\text { DIVORCE }\end{array}$ & $\begin{array}{c}\text { ÂGE MOYEN } \\
\text { SORTIE } \\
\text { PROPRIÉTÉ }\end{array}$ \\
\hline $\begin{array}{l}\text { MARIÉS } \\
\text { IDIVORCÉS }\end{array}$ & 23,7 & 32,3 & 34,1 & 35,7 & 35,8 \\
\hline HOMMES & 25,0 & 33,5 & 34,9 & 36,1 & 36,7 \\
\hline FEMMES & 22,6 & 31,4 & 33,5 & 35,4 & 35,1 \\
\hline $\begin{array}{l}\text { GÉNÉRATION } \\
\text { I930-1939 }\end{array}$ & 23,6 & 33,6 & 33,4 & 35,9 & 35,3 \\
\hline $\begin{array}{l}\text { GÉNÉRATION } \\
\text { I940-I945 }\end{array}$ & 23,5 & 32,0 & 35,1 & 36,5 & 35,0 \\
\hline $\begin{array}{l}\text { GÉNÉRATION } \\
\text { I946-I950 }\end{array}$ & 23,8 & 31,8 & 33,7 & 35,0 & 36,4 \\
\hline
\end{tabular}

Source: Enquête «Biographies et entourage», INED, 200I.

9 Les divorcés se distinguent des autres couples par un âge au mariage plus précoce, (23,7 ans contre 25, I). 
Le divorce contraint les femmes dans un certain nombre de cas à quitter le statut de propriétaire, ce qui s'explique notamment par des ressources moindres qui ne leur permettent pas de faire face au rachat du logement et aux remboursements des mensualités. La propriété qui avait été choisie en partie parce qu'elle devait assurer un toit à la famille ne joue pas dans la majorité des cas son rôle protecteur. Et le statut qui favorise le plus la stabilité résidentielle après la rupture conjugale s'avère être celui de locataire dans le parc social (36\% des locataires HLM n'ont pas déménagé contre $25 \%$ des propriétaires (Clément et Bonvalet, 2006). Depuis les travaux de Patrick Festy en 1988, on sait que l'après-divorce peut être pour certains une période de fortes instabilités résidentielles avec des retours chez les parents, des séjours à l'hôtel ou dans des locations meublées.

C'est d'autant plus vrai pour les hommes qui, d’après les entretiens menés auprès des personnes sélectionnées dans l'enquête «Biographies et entourage $\gg^{\circ}$, connaissent plus souvent des étapes résidentielles composées de logements temporaires. Ayant, dans la majorité des cas, la garde des enfants, les femmes auront plus facilement accès au logement social où elles sont considérées comme prioritaires. En effet, comme l'ont montré les travaux de Pascale Dietrich-Ragon (2013), les personnes seules et célibataires bénéficient moins de prises en charge institutionnelle. Aussi n'est-il pas surprenant de constater que, d'après les résultats d'un modèle de Cox, une rupture conjugale augmente de manière très significative le fait d'entrer en HLM pour les femmes (risque instantané multiplié par 2, p<o,oI), alors qu'il n’augmente que de $35 \%$ chez les hommes $(\mathrm{p}<\mathrm{0}, \mathrm{I} \mathrm{O})$. Pour les femmes de milieux populaires, toutes choses égales par ailleurs, le logement social joue un rôle très protecteur. Il leur permet notamment de se maintenir dans les lieux quand le lien conjugal se délite et s'avère plus accessible après une séparation ou un divorce.

I0 Voir l'analyse des entretiens de Céline Clément dans la recherche sur les espaces de vie des familles recomposées (Clément et Bonvalet, 2006, p. 50). 
Tableau 5. Modèle de Cox, impact sur l'accès au logement HLM après une rupture

\begin{tabular}{|c|c|c|c|}
\hline & & HOMME & FEMME \\
\hline \multirow{7}{*}{ DIPLÔME } & Aucun Diplôme & $2.342 * * * *$ & $2.772 * * * *$ \\
\hline & Inférieur au Bac & |.87| ${ }^{*} * * *$ & $2.055 * * * *$ \\
\hline & $\mathrm{Bac}$ & I (Réf) & I (Réf) \\
\hline & Inférieur à bac+3 & Ns & Ns \\
\hline & Licence, Maitrise & Ns & Ns \\
\hline & Capes, Dea, agrégation & $0.512 *$ & $0.412 *$ \\
\hline & Écoles Ingénieur & $0.124 * * * * *$ & Ns \\
\hline DIVORCE & divorce & 1.355* & $2.038 * * *$ \\
\hline \multirow{4}{*}{ GÉNÉRATION } & $1930-1934$ & I (Réf) & I (Réf) \\
\hline & $1935-1939$ & Ns & Ns \\
\hline & $1940-1945$ & Ns & Ns \\
\hline & $1946-1950$ & Ns & 1.853**** \\
\hline \multirow{4}{*}{ ORIGINE } & Paris & Ns & Ns \\
\hline & Banlieue & Ns & 1.486**⿻丷木 \\
\hline & Province & I (Réf) & I (Réf) \\
\hline & Autres & 1.460*** & $1.327 *$ \\
\hline
\end{tabular}

****significatif au seuil de $1 \%$;

**significatif au seuil de $5 \%$;

*Significatif au seuil de 10\%.

\section{CONCLUSION}

Au moment de la constitution de la famille, les couples vont rechercher la stabilité résidentielle en accédant au logement social ou à la propriété. Cette dernière répond au besoin de sécurité, à celui d’avoir un toit, au désir d’accumuler un patrimoine qui sera transmis plus tard aux enfants. Elle répond aux aspirations de création de maisonnée (Bourdieu, 20ı0). À défaut d’acheter une résidence principale, les couples trouveront une sécurité autre en accédant au logement HLM qui leur garantit le maintien dans les lieux. On retrouve là la notion de propriété sociale comme alternative possible à la propriété privée de Robert Castel (1999 [1996]). L'achat d'un logement ou l'obtention d'un loge- 
ment social intervient quelques années seulement après l'arrivée du premier ou deuxième enfant, confirmant les liens entre trajectoires familiales et trajectoires résidentielles. La stabilité résidentielle que procure la propriété a de fortes chances de durer tant que le couple résiste à l'épreuve du temps. En revanche, elle apparaît très compromise lorsque les conjoints se séparent. Si les femmes connaissent un risque de sortie de la propriété supérieur à celui des hommes, leur chance d’obtenir un logement social est nettement plus élevée. Quelles que soient les générations, les séparations et les divorces ont entraîné des bifurcations dans les «parcours logement» souvent brutales en interrompant des parcours ascendants en termes de position résidentielle où le statut de propriétaire est considéré comme un signe de réussite sociale. Alors que les trajectoires résidentielles des générations nées avant guerre, à l’instar de leurs trajectoires familiales, se déroulaient de manière linéaire selon un schéma allant de la location vers la propriété, les trajectoires des générations nées après 1945 apparaissent nettement plus chaotiques, au gré des unions, séparations et remises en couple éventuelles. Le poids des séparations et des divorces dans des «parcours logement» de plus en plus complexes est tel qu'il est l'une des raisons de la moindre croissance de la proportion de propriétaires parmi les générations de Franciliens nées après guerre ainsi qu'une partie accrue de la demande de logements sociaux.

\section{BIBLIOGRAPHIE}

BERGER M., 2004 , Les Périurbains de Paris. De la ville dense à la ville éclatée, Paris, CNRS Éditions.

BONVALET C., 1998, «Accession a la propriété et trajectoires individuelles», in Trajectoires familiales et espace de vie en milieu urbain, Y. Grafmeyer,

F. Dansereau (dir.), Lyon, PUL, p. 235-262.

-., 2007, «Histoire résidentielle d'une génération», in Les Stratégies résidentielles et patrimoniales: vieillissement de la population et logement, Bonvalet et al. (dir.), Paris, La Documentation française, p. 283-302. 
BONVALET C. et BRINGÉ A., 2013, «Les effets de la politique de logement sur l'évolution des taux de propriétaires en France», Revue européenne des sciences sociales, 5I-I, p. I53-177.

BONVALET C. et MAISON D., 1996, «Lieux d'ancrage et lieux de passage: itinéraires résidentiels en région parisienne», in La Ville: agrégation et ségrégation sociales, N. Haumont (dir.), Paris, L'Harmattan, p. 173-187.

BONVALET C. et MERLIN P. (dir.), 1988, Les Transformations de la famille et l'habitat: bibliographie commentée, Paris, La Documentation française.

BONVALET C. et TUGAULTY., 1984, «Les racines du dépeuplement de Paris », Population, 3, p.463-482.

BONVALET C., CLÉMENT C. et OGG J., 20II, Réinventer la famille. L'histoire des baby-boomers, Paris, PUF.

BOURDIEU P., 1990, «Un placement de père de famille: la maison individuelle: spécificité du produit et logique du champ de production»», Annales de la Recherche en Sciences Sociales, «L'économie de la maison», 8I/82, p.6-33.

-, 2000, Les Structures sociales de l'économie, Paris, Éditions du Seuil.

CASTEL R., 1999 (1996), Les Métamorphoses de la question sociale, Paris, Gallimard, «Folio essais».

CLÉMENT C et BONVALET C., 2006, Les Espaces de vie des familles recomposées, Paris, PUCA.

DIETRICH-RAGON P., 2013, «Qui rêve du logement social?», Sociologie, I-4, [en ligne]: $<$ http://sociologie.revues.org//512>.

FESTY P., 1990, «Mobilité résidentielle des femmes séparées: une étape dans le cycle familial» in Stratégies residentielles, C. Bonvalet, A. M. Fribourg (dir.), Paris; INED, Congrès et Colloques, 2, p. 231-263.

GENGEMBRE G.,1989, «La famille des contre-révolutionnaires: une réponse archaïque à la modernité», in La Famille, la Loi, l'État: de la Révolution au Code civil, l. Théry, et C. Biet, Paris, CNRS, Centre Georges Pompidou, Imprimerie Nationale.

GRAFMEYER Y., 1994, Sociologie urbaine, Paris, Nathan. 
IMBERT C., 2012, «La diversité des ancrages des populations passées par les villes nouvelles», in De la famille à l'entourage. L'enquête biographies et entourage, C. Bonvalet et É. Lelièvre, Paris, INED, p. 289-30I.

LOISEAU M. et BONVALET C ., 2005, «Effet à long terme d'une politique de régulation des loyers: rôle du parc de logements soumis à la loi de 1948», Population, 3, p. 35I-366.

RAYMOND H., RAYMOND M. G et HAUMONT N., 1966, Les Pavillonnaires, Paris, Centre de Recherche d'Urbanisme.

SEGALEN M., 2010, Sociologie de la famille, Paris, Armand Colin.

TOPALOV C., 1987, Le Logement en France. Histoire d'une marchandise impossible, Paris, Presses de la Fondation nationale des sciences politiques.

TOULEMON L., 1994, «Les étapes vers l'âge adulte: vers un nouveau statut des femmes», in Constances et inconstances de la famille, $\mathrm{H}$. Léridon et C. Villeneuve-Gokalp (dir.), Paris, INED, p. 165-181. 